\title{
Positivity of sums and integrals for $n$-convex functions via the Fink identity and new Green functions
}

\author{
Asif R. Khan and Josip Pečarić
}

\begin{abstract}
We consider positivity of sum $\sum_{i=1}^{n} p_{i} f\left(x_{i}\right)$ involving convex functions of higher order. Analogous for integral $\int_{a}^{b} p(x) f(g(x)) d x$ is also given. Representation of a function $f$ via the Fink identity and the Green function leads us to identities for which we obtain conditions for positivity of the mentioned sum and integral. We obtain bounds for integral remainders which occur in those identities as well as corresponding mean value theorems.
\end{abstract}

Mathematics Subject Classification (2010): 26A51, 26D15, 26D20.

Keywords: $n$-convex functions, Fink identity, Green function, Čebyšev functional.

\section{Introduction}

In [9] we proved various results related to general linear inequalities via Fink identity with and without Green function (see also [8]). Recently, in [2] the authors have introduced new Green type functions. Our main objective of present article is to further extend results of [9] using new definitions stated in [2].

To recall the definitions of generalized convex function and related concepts and results we refer to interested readers the following references [10], [5] and [15].

In the sequel we use the notation $A C[a, b]$ for class of absolutely continuous functions defined on a real interval $[a, b]$ and by $(\xi-s)_{+}^{k}, k \in \mathbb{N}_{0}$, we will mean the following

$$
(\xi-s)_{+}^{k}= \begin{cases}(\xi-s)^{k}, & \text { if } \xi \geq s \\ 0, & \text { if } \xi<s .\end{cases}
$$

Now we recall the Fink identity to prove many interesting results. The following theorem is proved by A. M. Fink in [4]. 
Proposition 1.1. Let $a, b \in \mathbb{R}, f:[a, b] \rightarrow \mathbb{R}, n \geq 1$ and $f^{(n-1)}$ is absolutely continuous on $[a, b]$. Then

$$
\begin{aligned}
f(x) & =\frac{n}{b-a} \int_{a}^{b} f(t) d t-\sum_{k=1}^{n-1} \frac{n-k}{k !}\left(\frac{f^{(k-1)}(a)(x-a)^{k}-f^{(k-1)}(b)(x-b)^{k}}{b-a}\right) \\
& +\frac{1}{(n-3) !(b-a)} \int_{a}^{b}(x-t)^{n-1} P^{[a, b]}(t, x) f^{(n)}(t) d t,
\end{aligned}
$$

where

$$
P^{[a, b]}(t, x)= \begin{cases}t-a, & a \leq t \leq x \leq b \\ t-b, & a \leq x<t \leq b\end{cases}
$$

Pečarić in [12] proved the following result (see also [15, p.262]):

Proposition 1.2. The inequality

$$
\sum_{i=1}^{m} p_{i} f\left(x_{i}\right) \geq 0
$$

holds for all convex functions $f$ if and only if the $m$-tuples

$$
\mathbf{x}=\left(x_{1}, \ldots, x_{m}\right), \quad \mathbf{p}=\left(p_{1}, \ldots, p_{m}\right) \in \mathbb{R}^{m}
$$

satisfy

$$
\sum_{i=1}^{m} p_{i}=0 \quad \text { and } \quad \sum_{i=1}^{m} p_{i}\left|x_{i}-x_{k}\right| \geq 0 \text { for } k \in\{1, \ldots, m\} .
$$

Since

$$
\sum_{i=1}^{m} p_{i}\left|x_{i}-x_{k}\right|=2 \sum_{i=1}^{m} p_{i}\left(x_{i}-x_{k}\right)_{+}-\sum_{i=1}^{m} p_{i}\left(x_{i}-x_{k}\right),
$$

where $y_{+}=\max (y, 0)$, it is easy to see that condition (1.4) is equivalent to

$$
\sum_{i=1}^{m} p_{i}=0, \quad \sum_{i=1}^{m} p_{i} x_{i}=0 \quad \text { and } \quad \sum_{i=1}^{m} p_{i}\left(x_{i}-x_{k}\right)_{+} \geq 0 \text { for } k \in\{1, \ldots, m-1\} .
$$

The following result is due to Popoviciu [16, 17] (see [15, 18] also).

Proposition 1.3. Let $n \geq 2$. Inequality (1.3) holds for all $n$-convex functions $f$ : $[a, b] \rightarrow \mathbb{R}$ if and only if the $m$-tuples $\mathbf{x} \in[a, b]^{m}, \mathbf{p} \in \mathbb{R}^{m}$ satisfy

$$
\begin{aligned}
& \sum_{i=1}^{m} p_{i} x_{i}^{k}=0, \quad \text { for all } k \in\{0,1, \ldots, n-1\} \\
& \sum_{i=1}^{m} p_{i}\left(x_{i}-t\right)_{+}^{n-1} \geq 0, \quad \text { for every } t \in[a, b] .
\end{aligned}
$$

Proposition 1.4. Let $n \geq 2, p:[\alpha, \beta] \rightarrow \mathbb{R}$ and $g:[\alpha, \beta] \rightarrow[a, b]$. Then, the inequality

$$
\int_{\alpha}^{\beta} p(x) f(g(x)) d x \geq 0
$$


holds for all $n$-convex functions $f:[a, b] \rightarrow \mathbb{R}$ if and only if

$$
\begin{aligned}
& \int_{\alpha}^{\beta} p(x) g(x)^{k} d x=0, \quad \text { for all } k \in\{0,1, \ldots, n-1\} \\
& \int_{\alpha}^{\beta} p(x)(g(x)-t)_{+}^{n-1} d x \geq 0, \quad \text { for every } t \in[a, b] .
\end{aligned}
$$

After this introductory section, we continue with section 2 where identities for

$$
\sum_{i=1}^{n} p_{i} f\left(x_{i}\right) \text { and } \int_{a}^{b} p(x) f(g(x)) d x
$$

are given using the Fink identity and new Green functions. Also we consider inequalities for $n$-convex functions which are based on these identities. Section 3 is devoted to estimations of functions $A_{k}$ by using Čebyšev, Gr̈uss and Ostrowski type inequalities and the Hölder inequality. In the last section we give mean value theorems for functionals $A_{k}, k \in\{1,2\}$.

\section{Popoviciu type identities and inequalities via the Fink identity and new Green functions}

In this section we obtain some discrete and integral identities and the corresponding linear inequalities using new Green functions and applying the Fink identity. As a special choice of Abel-Gontscharoff polynomial for 'two-point right focal' interpolating polynomial for $n=2$ could be stated as (see [13]):

$$
f(\xi)=f(a)+(\xi-a) f^{\prime}(b)+\int_{a}^{b} G_{1}(\xi, t) f^{\prime \prime}(t) d t,
$$

where $G_{1}(s, t)$ is Green's function for 'two-point right focal problem' defined as

$$
G_{1}(s, t)= \begin{cases}a-t, & a \leq t \leq s \\ a-s, & s \leq t \leq b\end{cases}
$$

Motivated by Abel-Gontscharoff identity (2.1) and related Green's function (2.2), we recall some new types of Green functions $G_{l}:[a, b] \times[a, b] \rightarrow \mathbb{R},(l=2,3,4$,$) defined$ as in $[2]$ :

$$
\begin{aligned}
& G_{2}(s, t)= \begin{cases}s-b, & a \leq t \leq s, \\
t-b, & s \leq t \leq b .\end{cases} \\
& G_{3}(s, t)= \begin{cases}s-a, & a \leq t \leq s, \\
t-a, & s \leq t \leq b .\end{cases} \\
& G_{4}(s, t)= \begin{cases}b-t, & a \leq t \leq s, \\
b-s, & s \leq t \leq b .\end{cases}
\end{aligned}
$$

In [2], it is also shown that all four Green functions are symmetric and continuous. Moreover, all functions are convex with respect to both variables $s$ and $t$. From these functions we can obtain new identities, given in following lemma: 
Lemma 2.1. Let $f:[a, b] \rightarrow \mathbb{R}$ be twice differentiable function and $G_{l},(l=2,3,4)$ are defined in (2.3), (2.4) and (2.5). Then the following identities holds:

$$
\begin{aligned}
& f(\xi)=f(b)+(b-\xi) f^{\prime}(a)+\int_{a}^{b} G_{2}(\xi, t) f^{\prime \prime}(t) d t \\
& f(\xi)=f(b)-(b-a) f^{\prime}(b)+(\xi-a) f^{\prime}(a)+\int_{a}^{b} G_{3}(\xi, t) f^{\prime \prime}(t) d t, \\
& f(\xi)=f(a)+(b-a) f^{\prime}(a)-(b-\xi) f^{\prime}(b)+\int_{a}^{b} G_{4}(\xi, t) f^{\prime \prime}(t) d t .
\end{aligned}
$$

We can easily obtain these identities by using integration by parts by using respective Green function. Now we state here main results related to the Fink identity and the Green function.

Theorem 2.2. Fix $l \in\{1,2,3,4\}$. Let $f:[a, b] \rightarrow \mathbb{R}$ be such that for $n \geq 3$, $f^{(n-1)}$ is absolutely continuous. Let $x_{i}, y_{i} \in[a, b], p_{i} \in \mathbb{R}$ for $i \in\{1, \ldots, m\}$ be such that $\sum_{i=1}^{m} p_{i}=0$ and $\sum_{i=1}^{m} p_{i} x_{i}=0$ and let $P^{[a, b]}(t, x)$ be the same as defined in (1.2). If $G_{l}$ are the Green functions as defined in $(2.2)-(2.5)$, then we have

$$
\begin{aligned}
& \sum_{i=1}^{m} p_{i} f\left(x_{i}\right)=\sum_{k=0}^{n-3}\left(\frac{n-k-2}{k !(b-a)}\right) \int_{a}^{b}\left(\sum_{i=1}^{m} p_{i} G_{l}\left(x_{i}, s\right)\right) \\
& \times\left(f^{(k+1)}(b)(s-b)^{k}-f^{(k+1)}(a)(s-a)^{k}\right) d s+\frac{1}{(n-3) !(b-a)} \\
& \times \int_{a}^{b} f^{(n)}(t)\left(\int_{a}^{b} \sum_{i=1}^{m} p_{i} G_{l}\left(x_{i}, s\right)(s-t)^{n-3} P^{[a, b]}(t, s) d s\right) d t .
\end{aligned}
$$

Proof. First consider four identities $(2.1),(2.6),(2.7)$ and $(2.8)$, and putting $x=\xi_{i}$ in all these identities, multiplying each with $p_{i}$, and then summing over each identity for $i \in\{1, \ldots, m\}$ and using conditions that $\sum_{i=1}^{m} p_{i}=0, \sum_{i=1}^{m} p_{i} \xi_{i}=0$ we get by fixing $l \in\{1,2,3,4\}$

$$
\sum_{i=1}^{m} p_{i} f\left(\xi_{i}\right)=\int_{a}^{b}\left(\sum_{i=1}^{m} p_{i} G_{l}\left(\xi_{i}, t\right)\right) f^{\prime \prime}(t) d t .
$$

Differentiating Fink identity twice we easily get

$$
\begin{aligned}
f^{\prime \prime}(x) & =\sum_{k=0}^{n-3} \frac{n-k-2}{k !} \frac{f^{(k+1)}(b)(x-b)^{k}-f^{(k+1)}(a)(x-a)^{k}}{b-a} \\
& +\frac{1}{(n-3) !(b-a)} \int_{a}^{b}(x-t)^{n-3} P^{[a, b]}(t, x) f^{(n)}(t) d t,
\end{aligned}
$$


and by using (2.11) in (2.10), we have

$$
\begin{aligned}
& \sum_{i=1}^{m} p_{i} f\left(x_{i}\right)=\int_{a}^{b}\left(\sum_{i=1}^{m} p_{i} G_{l}\left(x_{i}, s\right)\right) \\
& \times \sum_{k=0}^{n-3} \frac{n-k-2}{k !} \frac{f^{(k+1)}(b)(s-b)^{k}-f^{(k+1)}(a)(s-a)^{k}}{b-a} d s \\
& +\frac{1}{(n-3) !(b-a)} \int_{a}^{b} \sum_{i=1}^{m} p_{i} G_{l}\left(x_{i}, s\right)\left(\int_{a}^{b}(s-t)^{n-3} P^{[a, b]}(t, s) f^{(n)}(t) d t\right) d s .
\end{aligned}
$$

Now by interchanging the integral and summation in the second term and by applying Fubini's theorem in the last term, we have (2.9).

The following theorem is the integral version of Theorem 2.2.

Theorem 2.3. Fix $l \in\{1,2,3,4\}$. Let $f:[a, b] \rightarrow \mathbb{R}$ be such that for $n \geq 3$, $f^{(n-1)}$ is absolutely continuous on $[a, b]$ and let $p:[\alpha, \beta] \rightarrow \mathbb{R}$ and $g:[\alpha, \beta] \rightarrow[a, b]$ be integrable functions such that $\int_{\alpha}^{\beta} p(x) d x=0$ and $\int_{\alpha}^{\beta} p(x) g(x) d x=0$. Let $P^{[a, b]}(t, x)$ be the same as defined in (1.2). If $G_{l}$ are the Green functions as defined in $(2.2)-(2.5)$, then we have

$$
\begin{aligned}
& \int_{\alpha}^{\beta} p(x) f(g(x)) d x=\sum_{k=0}^{n-3} \frac{n-k-2}{k !(b-a)} \int_{a}^{b}\left(\int_{\alpha}^{\beta} p(x) G_{l}(g(x), s) d x\right) \\
& \left(f^{(k+1)}(b)(s-b)^{k}-f^{(k+1)}(a)(s-a)^{k}\right) d s+\frac{1}{(n-3) !(b-a)} \\
& \times \int_{a}^{b} f^{(n)}(t)\left(\int_{a}^{b}\left(\int_{\alpha}^{\beta} p(x) G_{l}(g(x), s) d x\right)(s-t)^{n-3} P^{[a, b]}(t, s) d s\right) d t .
\end{aligned}
$$

Proof. Since the proof is similar to that of the previous theorem, we omit the details.

Here we introduce some notations which will be used in rest of the paper:

$$
\begin{aligned}
\Omega_{1}^{[a, b]}(m, \mathbf{x}, \mathbf{p}, t) & =\int_{a}^{b} \sum_{i=1}^{m} p_{i} G_{l}\left(x_{i}, s\right)(s-t)^{n-3} P^{[a, b]}(t, s) d s \\
\Omega_{2}^{[a, b]}([\alpha, \beta], g, p, t) & =\int_{a}^{b} \int_{\alpha}^{\beta} p(x) G_{l}(g(x), s) d x(s-t)^{n-3} P^{[a, b]}(t, s) d s .
\end{aligned}
$$




$$
\begin{aligned}
A_{1}^{[a, b]}(m, \mathbf{x}, \mathbf{p}, f) & =\sum_{i=1}^{m} p_{i} f\left(x_{i}\right)-\sum_{k=0}^{n-3}\left(\frac{n-k-2}{k !(b-a)}\right) \int_{a}^{b} \sum_{i=1}^{m} p_{i} G_{l}\left(x_{i}, s\right) \\
& \times\left(f^{(k+1)}(b)(s-b)^{k}-f^{(k+1)}(a)(s-a)^{k}\right) d s \\
A_{2}^{[a, b]}([\alpha, \beta], g, p, f) & =\int_{\alpha}^{\beta} p(x) f(g(x)) d x \\
& -\sum_{k=0}^{n-3}\left(\frac{n-k-2}{k !(b-a)}\right) \int_{a}^{b}\left(\int_{\alpha}^{\beta} p(x) G_{l}(g(x), s) d x\right) \\
& \times\left(f^{(k+1)}(b)(s-b)^{k}-f^{(k+1)}(a)(s-a)^{k}\right) d s .
\end{aligned}
$$

The following theorem is our second main result of this section:

Theorem 2.4. Let all the assumptions of Theorem 2.2 be satisfied and let for $n \geq 3$, the inequality

$$
\Omega_{1}^{[a, b]}(m, \mathbf{x}, \mathbf{p}, t) \geq 0
$$

holds. If $f$ is n-convex, then we have

$$
A_{1}^{[a, b]}(m, \mathbf{x}, \mathbf{p}, f) \geq 0 .
$$

If opposite inequality holds in (2.17), then (2.18) holds in the reverse direction.

Proof. Since $f^{(n-1)}$ is absolutely continuous on $[a, b], f^{(n)}$ exists almost everywhere. As $f$ is $n$-convex, applying definition, we have, $f^{(n)}(x) \geq 0$ for all $x \in[a, b]$. Now by using $f^{(n)} \geq 0$ and (2.17) in (2.9), we have (2.18).

Corollary 2.5. Let all the assumptions of Theorem 2.2 be satisfied. In addition we let

$$
\sum_{i=1}^{m} p_{i}\left(x_{i}-x_{k}\right)_{+} \geq 0 \quad \text { for } \quad k \in\{1, \ldots, m\} .
$$

Let $n$ be even and $n>3$. If the function $f:[a, b] \rightarrow \mathbb{R}$ is $n$-convex, then inequality (2.18) is satisfied, $i$. e.

$$
\begin{aligned}
& \sum_{i=1}^{m} p_{i} f\left(x_{i}\right) \geq \sum_{k=0}^{n-3} \frac{n-k-2}{k !(b-a)} \int_{a}^{b} \sum_{i=1}^{m} p_{i} G_{l}\left(x_{i}, s\right) \\
& \times\left(f^{(k+1)}(b)(s-b)^{k}-f^{(k+1)}(a)(s-a)^{k}\right) d s .
\end{aligned}
$$

Further if $f^{(k+1)}(a) \leq 0$ and $(-1)^{k} f^{(k+1)}(b) \geq 0$ for $k \in\{0,1, \ldots, n-3\}$ then

$$
\sum_{i=1}^{m} p_{i} f\left(x_{i}\right) \geq 0 \text {. }
$$

Proof. We fix $l \in\{1,2,3,4\}$ and $n>3$. As $\mathbf{x}$ and $\mathbf{p}$ are real $m$-tuples such that they satisfy the assumption (1.5), by using the convex function $x \mapsto G_{l}(x, s)$ in (1.3), we obtain

$$
\sum_{i=1}^{m} p_{i} G_{l}\left(x_{i}, s\right) \geq 0 .
$$


For $a \leq s \leq t$, it is easy to see that

$$
\int_{a}^{t} \sum_{i=1}^{m} p_{i} G_{l}\left(x_{i}, s\right)(s-t)^{n-3} P^{[a, b]}(t, s) d s \geq 0
$$

holds for even $n$. Now as $f$ is $n$-convex for even $n$, by applying Theorem 2.4 , we get (2.19).

If $a \leq s \leq b$ and $k \in\{0, \ldots, n-3\}$, then from assumptions $f^{(k+1)}(a) \leq 0$ and $(-1)^{k} f^{(k+1)}(b) \geq 0$ we have that

$$
f^{(k+1)}(b)(s-b)^{k}-f^{(k+1)}(a)(s-a)^{k} \geq 0,
$$

So, from inequalities (2.19), (2.20) and (2.22) the non-negativity of the right hand side of (2.19) is immediate.

An integral version of our second main result states that:

Theorem 2.6. Let all the assumptions of Theorem 2.3 be satisfied and let for $n \geq 3$, the inequality

$$
\Omega_{2}^{[a, b]}([\alpha, \beta], g, p, t) \geq 0
$$

holds. If $f$ is n-convex, then we have

$$
A_{2}^{[a, b]}([\alpha, \beta], g, p, f) \geq 0 .
$$

If opposite inequality holds in (2.14), then (2.24) holds in the reverse direction.

Proof. The idea of the proof is the same as that of the proof of Theorem 2.4. By using $f^{(n)} \geq 0$ and (2.14) in (2.12), we have (2.24).

Corollary 2.7. Let all the assumptions of Theorem 2.3 be satisfied. In addition we let

$$
\int_{\alpha}^{\beta} p(x)(g(x)-t)_{+}^{n-1} d x \geq 0, \quad \text { for every } t \in[a, b] .
$$

Let $n$ be even and $n>3$. If the function $f:[a, b] \rightarrow \mathbb{R}$ is $n$-convex, then we have

$$
\begin{aligned}
& \int_{\alpha}^{\beta} p(x) f(g(x)) d x \geq \sum_{k=0}^{n-3} \frac{n-k-2}{k !(b-a)} \int_{a}^{b}\left(\int_{\alpha}^{\beta} p(x) G_{l}(g(x), s) d x\right) \\
& \times\left(f^{(k+1)}(b)(s-b)^{k}-f^{(k+1)}(a)(s-a)^{k}\right) d s .
\end{aligned}
$$

Further if $f^{(k+1)}(a) \leq 0$ and $(-1)^{k} f^{(k+1)}(b) \geq 0$ for $k \in\{0,1 \ldots, n-3\}$, then the right hand side of (2.25) is non-negative.

Proof. The proof is analogous to the proof of Corollary 2.5 but instead of Theorem 2.4, we apply Theorem 2.6. 


\section{Related inequalities for $n$-convex functions at a point}

In this section we will give related results for the class of $n$-convex functions at a point introduced in [14].

Definition 3.1. Let $I$ be an interval in $\mathbb{R}, c$ a point in the interior of $I$ and $n \in \mathbb{N}$. A function $f: I \rightarrow \mathbb{R}$ is said to be $n$-convex at point $c$ if there exists a constant $K$ such that the function

$$
F(x)=f(x)-\frac{K}{(n-3) !} x^{n-1}
$$

is $(n-1)$-concave on $I \cap(-\infty, c]$ and $(n-1)$-convex on $I \cap[c, \infty)$. A function $f$ is said to be $n$-concave at point $c$ if the function $-f$ is $n$-convex at point $c$.

Let $e_{i}$ denote the monomials $e_{i}(x)=x^{i}, i \in \mathbb{N}_{0}$. First we state main results for discrete case.

Theorem 3.2. Let $c \in(a, b), \mathbf{x} \in[a, c]^{m}, \mathbf{y} \in[c, b]^{l}, \mathbf{p} \in \mathbb{R}^{m}, \mathbf{q} \in \mathbb{R}^{l}$ and $f$ : $[a, b] \rightarrow \mathbb{R}$ be a function such that $f^{(n-1)}$ is absolutely continuous. Let $\Omega_{1}^{[\cdot, \cdot]}(\cdot, \cdot, \cdot, t)$ and $A_{1}^{[\cdot, \cdot]}(\cdot, \cdot, \cdot, f)$ be defined as in (2.13) and (2.15) and satisfy the following conditions:

$$
\begin{array}{cll}
\Omega_{1}^{[a, c]}(m, \mathbf{x}, \mathbf{p}, t) \geq 0 & \text { for every } & t \in[a, c], \\
\Omega_{1}^{[c, b]}(l, \mathbf{y}, \mathbf{q}, t) \geq 0 & \text { for every } & t \in[c, b],
\end{array}
$$

and

$$
A_{1}^{[a, c]}\left(m, \mathbf{x}, \mathbf{p}, e_{n}\right)=A_{1}^{[c, b]}\left(l, \mathbf{y}, \mathbf{q}, e_{n}\right) .
$$

If $f$ is $(n+1)$-convex at point $c$, then

$$
A_{1}^{[a, c]}(m, \mathbf{x}, \mathbf{p}, f) \leq A_{1}^{[c, b]}(l, \mathbf{y}, \mathbf{q}, f) .
$$

If inequalities in (3.1) and (3.2) are reversed, then (3.4) holds with the reverse sign of inequality.

Proof. Let $F=f-\frac{K}{n !} e_{n}$ be as in Definition 3.1, i. e., the function $F$ is $n$-concave on $[a, c]$ and $n$-convex on $[c, b]$. Applying Theorem 2.4 to $F$ on the interval $[a, c]$ and on the interval $[c, b]$ we have

$$
A_{1}^{[a, c]}(m, \mathbf{x}, \mathbf{p}, F) \leq 0 \leq A_{1}^{[c, b]}(l, \mathbf{y}, \mathbf{q}, F) .
$$

Using definition of $F$ we obtain

$$
A_{1}^{[a, c]}(m, \mathbf{x}, \mathbf{p}, f)-\frac{K}{n !} A_{1}^{[a, c]}\left(m, \mathbf{x}, \mathbf{p}, e_{n}\right) \leq A_{1}^{[c, b]}(l, \mathbf{y}, \mathbf{q}, f)-\frac{K}{n !} A_{1}^{[c, b]}\left(l, \mathbf{y}, \mathbf{q}, e_{n}\right) .
$$

Since equality (3.3) is valid we get

$$
A_{1}^{[a, c]}(m, \mathbf{x}, \mathbf{p}, f) \leq A_{1}^{[c, b]}(l, \mathbf{y}, \mathbf{q}, f) .
$$

Remark 3.3. A closer look at the proof of Theorem 3.2 gives us that a similar result hold if instead equality (3.3) we consider a positivity of the difference

$$
K\left(A_{k}^{[c, b]}\left(l, \mathbf{y}, \mathbf{q}, e_{n}\right)-A_{k}^{[a, c]}\left(m, \mathbf{x}, \mathbf{p}, e_{n}\right)\right) \geq 0 .
$$


Corollary 3.4. Let $j_{1}, j_{2}, n \in \mathbb{N}, 2 \leq j_{1}, j_{2} \leq n$ and let $f:[a, b] \rightarrow \mathbb{R}$ be $(n+1)$-convex at point c. Let $m$-tuples $\mathbf{x} \in[a, c]^{m}$ and $\mathbf{p} \in \mathbb{R}^{m}$ satisfy (1.6) and (1.7) with $n$ replaced by $j_{1}$, let l-tuples $\mathbf{y} \in[c, b]^{l}$ and $\mathbf{q} \in \mathbb{R}^{l}$ satisfy

$$
\begin{gathered}
\sum_{i=1}^{l} q_{i} y_{i}^{k}=0, \quad \text { for all } k=0,1, \ldots, j_{2}-1 \\
\sum_{i=1}^{l} q_{i}\left(y_{i}-t\right)_{+}^{j_{2}-1} \geq 0, \quad \text { for every } t \in\left[y_{(1)}, y_{(l-n+1)}\right]
\end{gathered}
$$

and let (3.3) holds. If $n-j_{1}$ and $n-j_{2}$ are even, then (3.4) holds.

Remark 3.5. For idea of the proof see [8, pp. 171-172].

Integral analogous of previous theorem may be stated as:

Theorem 3.6. Let $c \in(a, b)$ and let $g:[\alpha, \beta] \rightarrow[a, c], p:[\alpha, \beta] \rightarrow \mathbb{R}, h:[\gamma, \delta] \rightarrow[c, b]$, $q:[\gamma, \delta] \rightarrow \mathbb{R}$ be integrable functions. Let $f: I \rightarrow \mathbb{R},[a, b] \subset I$ be a function such that $f^{(n-1)}$ is absolutely continuous. Let $\Omega_{2}^{[\cdot, \cdot]}(\cdot, \cdot, \cdot, t)$ and $A_{2}^{[\cdot, \cdot]}(\cdot, \cdot, \cdot, f)$ be defined as in (2.14) and (2.16) satisfy the following conditions:

$$
\begin{aligned}
& \Omega_{2}^{[a, c]}([\alpha, \beta], g, p, t) \geq 0 \quad \text { for every } \quad t \in[a, c], \\
& \Omega_{2}^{[c, b]}([\gamma, \delta], h, q, t) \geq 0 \quad \text { for every } \quad t \in[c, b],
\end{aligned}
$$

and

$$
A_{2}^{[a, c]}\left([\alpha, \beta], g, p, e_{n}\right)=A_{2}^{[c, b]}\left([\gamma, \delta], h, q, e_{n}\right)
$$

If $f$ is $(n+1)$-convex at point $c$ (for $k=3, n \geq 3)$, then

$$
A_{2}^{[a, c]}([\alpha, \beta], g, p, f) \leq A_{2}^{[c, b]}([\gamma, \delta], h, q, f) .
$$

If inequalities in (3.5) and (3.6) are reversed, then (3.8) holds with the reverse sign of inequality.

Corollary 3.7. Let $j_{1}, j_{2}, n \in \mathbb{N}, 2 \leq j_{1}, j_{2} \leq n$ and let $f:[a, b] \rightarrow \mathbb{R}$ be $(n+1)$-convex at point c. Let integrable functions $g:[\alpha, \beta] \rightarrow[a, c], p:[\alpha, \beta] \rightarrow \mathbb{R}$ satisfy (1.9) with $n$ replaced by $j_{1}$, let $h:[\gamma, \delta] \rightarrow[c, b], q:[\gamma, \delta] \rightarrow \mathbb{R}$ satisfy

$$
\begin{aligned}
& \int_{\gamma}^{\delta} q(x) h(x)^{k} d x=0, \quad \text { for all } k \in\left\{0,1, \ldots, j_{2}-1\right\} \\
& \int_{\gamma}^{\delta} q(x)(h(x)-t)_{+}^{j_{2}-1} d x \geq 0, \quad \text { for every } t \in[c, b] .
\end{aligned}
$$

and let (3.7) holds. If $n-j_{1}$ and $n-j_{2}$ are even, then (3.4) holds. 


\section{Bounds for $A_{k}^{[\cdot, \cdot]}(\cdot, \cdot, \cdot, f)$ and $R_{n}^{k}$}

Let $f, h:[a, b] \rightarrow \mathbb{R}$ be two Lebesgue integrable functions. We consider the Cebyšev functional

$$
T(f, h)=\frac{1}{b-a} \int_{a}^{b} f(x) h(x) d x-\left(\frac{1}{b-a} \int_{a}^{b} f(x) d x\right)\left(\frac{1}{b-a} \int_{a}^{b} h(x) d x\right) .
$$

The following results can be found in [3]:

Proposition 4.1. Let $f:[a, b] \rightarrow \mathbb{R}$ be a Lebesgue integrable function and let $h:[a, b] \rightarrow$ $\mathbb{R}$ be an absolutely continuous function with $(\cdot-a)(b-\cdot)\left[h^{\prime}\right]^{2} \in L[a, b]$. Then we have the inequality

$$
|T(f, h)| \leq \frac{1}{\sqrt{2}}\left(\frac{1}{b-a}|T(f, f)| \int_{a}^{b}(x-a)(b-x)\left[h^{\prime}(x)\right]^{2} d x\right)^{1 / 2} .
$$

The constant $\frac{1}{\sqrt{2}}$ in (4.2) is the best possible.

Proposition 4.2. Let $h:[a, b] \rightarrow \mathbb{R}$ be a monotonic nondecreasing function and let $f:[a, b] \rightarrow \mathbb{R}$ be an absolutely continuous function such that $f^{\prime} \in L_{\infty}[a, b]$. Then we have the inequality

$$
|T(f, h)| \leq \frac{1}{2(b-a)}\left\|f^{\prime}\right\|_{\infty} \int_{a}^{b}(x-a)(b-x) d h(x) .
$$

The constant $\frac{1}{2}$ in (4.3) is the best possible.

We use the well-known Hölders inequality and bound for the Čebyšev functional $T(f, h)$. This bound is given in the following proposition in which the pre-Grüss inequality is given [11].

Proposition 4.3. Let $f, h:[a, b] \rightarrow \mathbb{R}$ be Lebesgue integrable functions such that $f h:$ $[a, b] \in L(a, b)$. If

then

$$
\gamma \leq h(x) \leq \Gamma \quad \text { for } \quad x \in[a, b]
$$

$$
|T(f, h)| \leq \frac{1}{2}(\Gamma-\gamma) \sqrt{T(f, f)}
$$

Now by using aforementioned results, we are going to obtain generalizations of the result proved in the previous section.

Remark 4.4. For the sake of brevity, in present and next sections at some places we will use the notations $A_{k}(f)=A_{k}^{[\cdot, \cdot]}(\cdot, \cdot, \cdot, \cdot f)$ and $\Omega_{k}(t)=\Omega_{k}^{[\cdot, \cdot]}(\cdot, \cdot, \cdot, t)$ for $k \in\{1,2\}$ as defined in Theorems 2.4 and 2.6.

Now, we are ready to state main results of this section:

Theorem 4.5. Let $f:[a, b] \rightarrow \mathbb{R}$ be such that $f^{(n)}$ is an absolutely continuous function for $n \in \mathbb{N}$ with $(.-a)(b-.)\left[f^{(n+1)}\right]^{2} \in L[a, b]$. Then it holds for $k \in\{1,2\}$

$$
A_{k}(f)=\frac{\left[f^{(n-1)}(b)-f^{(n-1)}(a)\right]}{(n-3) !(b-a)} \int_{a}^{b} \Omega_{k}(s) d s+R_{n}^{k}(f ; a, b)
$$


where the remainder $R_{n}^{k}(f ; a, b)$ satisfies the estimation

$$
\left|R_{n}^{k}(f ; a, b)\right| \leq \frac{1}{(n-3) !}\left(\frac{(b-a)}{2}\left|T\left(\Omega_{k}, \Omega_{k}\right) \int_{a}^{b}(s-a)(b-s)\left[f^{(n+1)}(s)\right]^{2} d s\right|\right)^{1 / 2} .
$$

Proof. Fix $k \in\{1,2\}$. If we apply Proposition 4.3 for $f \rightarrow \Omega_{k}$ and $h \rightarrow f^{(n)}$, then we obtain

$$
\begin{aligned}
& \left|\frac{1}{b-a} \int_{a}^{b} \Omega_{k}(t) f^{(n)}(t) d t-\left(\frac{1}{b-a} \int_{a}^{b} \Omega_{k}(t) d t\right)\left(\frac{1}{b-a} \int_{a}^{b} f^{(n)}(t) d t\right)\right| \\
& \leq \frac{1}{\sqrt{2}}\left(\frac{1}{b-a}\left|T\left(\Omega_{k}, \Omega_{k}\right)\right| \int_{a}^{b}(t-a)(b-t)\left[f^{(n+1)}(t)\right]^{2} d t\right)^{1 / 2} .
\end{aligned}
$$

Therefore we have

$$
\frac{1}{(n-3) !} \int_{a}^{b} \Omega_{k}(t) f^{(n)}(t) d t=\frac{\left[f^{(n-1)}(b)-f^{(n-1)}(a)\right]}{(n-3) !(b-a)} \int_{a}^{b} \Omega_{k}(t) d t+R_{n}^{k}(f ; a, b) .
$$

where $R_{n}^{k}(f ; a, b)$ satisfies inequality (4.5). Now from identities (2.9) and (2.12) for $k \in\{1,2\}$ respectively, we obtain (4.5).

By using Proposition 4.2 we obtain the following Grüss type inequality.

Theorem 4.6. Let $f:[a, b] \rightarrow \mathbb{R}$ be such that $f^{(n)}$ is an absolutely continuous function for $n \in \mathbb{N}$ with $(.-a)(b-.)\left[f^{(n+1)}\right]^{2} \in L[a, b]$ with $f^{(n+1)} \geq 0$ on $[a, b]$. Then we have the representation (4.5) and the remainder $R_{n}^{k}(f ; a, b)$ satisfies the following condition for $k \in\{1,2\}$,

$$
\begin{aligned}
\left|R_{n}^{k}(f ; a, b)\right| & \leq \frac{1}{(n-3) !}\left\|\Omega_{k}^{\prime}\right\|_{\infty}\left\{\frac{b-a}{2}\left[f^{(n-1)}(b)+f^{(n-1)}(a)\right]\right. \\
& \left.-\left[f^{(n-2)}(b)-f^{(n-2)}(a)\right]\right\} .
\end{aligned}
$$

Proof. Fix $k \in\{1,2\}$. If we apply Proposition 4.2 for $f \rightarrow \Omega_{k}$ and $h \rightarrow f^{(n)}$, then we obtain

$$
\begin{aligned}
& \left|\frac{1}{b-a} \int_{a}^{b} \Omega_{k}(t) f^{(n)}(t) d t-\left(\frac{1}{b-a} \int_{a}^{b} \Omega_{k}(t) d t\right)\left(\frac{1}{b-a} \int_{a}^{b} f^{(n)}(t) d t\right)\right| \\
& \leq \frac{1}{2(b-a)}\left\|\Omega_{k}^{\prime}\right\|_{\infty} \int_{a}^{b}(t-a)(b-t) f^{(n+1)}(t) d t .
\end{aligned}
$$

Since

$$
\begin{aligned}
\int_{a}^{b}(t-a)(b- & t) f^{(n+1)}(t) d t=\int_{a}^{b}(2 t-a-b) f^{(n)}(t) d t \\
& =(b-a)\left[f^{(n-1)}(b)+f^{(n-1)}(a)\right]-2\left[f^{(n-2)}(b)-f^{(n-2)}(a)\right] .
\end{aligned}
$$

Therefore, by using the identities (2.9) and (2.12) for $k \in\{1,2\}$ respectively and (4.7) we deduce (4.6). 
Theorem 4.7. For $k=1$ we assume that $\mathbf{x}$ and $\mathbf{p}$ satisfy the assumptions of Theorem 2.2 and for $k=2$ we assume that $x$ and $p$ satisfy the assumptions of Theorem 2.3. Let $k \in\{1,2\}$. Let $f: I \rightarrow \mathbb{R},[a, b] \subseteq I$, be such that $f^{(n)}$ is an absolutely continuous function and

$$
\gamma \leq f^{(n)}(x) \leq \Gamma \quad \text { for } \quad x \in[a, b]
$$

Then

$$
A_{k}(f)=\frac{\left[f^{(n-1)}(b)-f^{(n-1)}(a)\right]}{(n-3) !(b-a)} \int_{a}^{b} \Omega_{k}(t) d t+R_{n}^{k}(f ; a, b),
$$

where the remainder $R_{n}^{k}(f ; a, b)$ satisfies the estimation

$$
\left|R_{n}^{k}(f ; a, b)\right| \leq \frac{b-a}{2(n-3) !}(\Gamma-\gamma) \sqrt{T\left(\Omega_{k}, \Omega_{k}\right)} .
$$

Proof. Fix $k \in\{1,2\}$. Using definition of $A_{k}$ and result from the second section we have

$$
\begin{aligned}
A_{k}(f) & =\frac{1}{(n-3) !} \int_{a}^{b} f^{(n)}(t) \Omega_{k}(t) d t \\
& =\frac{1}{(n-3) !(b-a)} \int_{a}^{b} f^{(n)}(t) d t \int_{a}^{b} \Omega_{k}(t) d t+R_{n}^{k}(f ; a, b) \\
& =\frac{\left[f^{(n-1)}(b)-f^{(n-1)}(a)\right]}{(n-3) !(b-a)} \int_{a}^{b} \Omega_{k}(t) d t+R_{n}^{k}(f ; a, b)
\end{aligned}
$$

where

$$
R_{n}^{k}(f ; a, b)=\frac{1}{(n-3) !}\left(\int_{a}^{b} f^{(n)}(t) \Omega_{k}(t) d t-\frac{1}{b-a} \int_{a}^{b} f^{(n)}(s) d s \int_{a}^{b} \Omega_{k}(t) d t\right) .
$$

If we apply Proposition 4.3 for $f \rightarrow \Omega_{k}$ and $h \rightarrow f^{(n)}$, then we obtain

$$
\left|R_{n}^{k}(f ; a, b)\right|=\left|T\left(\Omega_{k}, f^{(n)}\right)\right| \leq \frac{b-a}{2(n-3) !}(\Gamma-\gamma) \sqrt{T\left(\Omega_{k}, \Omega_{k}\right)} .
$$

Theorem 4.8. Let $k \in\{1,2\}$. Let $(q, r)$ be a pair of conjugate exponents, that is, $1 \leq q, r \leq \infty, \frac{1}{q}+\frac{1}{r}=1$. Let $f^{(n)} \in L_{q}[a, b]$ for some $n \in \mathbb{N}, n>1$. Further, for $k=1$ we assume that $\mathbf{x}$ and $\mathbf{p}$ satisfy the assumptions of Theorem 2.2 and for $k=2$ we assume that $x$ and $p$ satisfy the assumptions of Theorem 2.3. Then we have

$$
\left|A_{k}(f)\right| \leq \frac{1}{(n-3) !}\left\|f^{(n)}\right\|_{q}\left\|\Omega_{k}\right\|_{r}
$$

The constant on the right hand side of (4.10) is sharp for $1<q \leq \infty$ and the best possible for $q=1$.

Proof. Fix $k \in\{1,2\}$. From definition of $A_{k}$ and results from the second section, applying the Hölder inequality we get

$$
\left|A_{k}(f)\right|=\left|\frac{1}{(n-3) !} \int_{a}^{b} f^{(n)}(t) \Omega_{k}(t) d t\right| \leq\left\|f^{(n)}\right\|_{q}\left\|\lambda_{k}\right\|_{r}
$$


where we denoted $\frac{1}{(n-3) !} \Omega_{k}$ by $\lambda_{k}$.

The sharpness of the constant $\left(\int_{a}^{b}\left|\lambda_{k}(t)\right|^{r} d s\right)^{1 / r}$ can be proved by considering the following function $f$ for which the equality in (4.10) is obtained.

For $1<q<\infty$ we take $f$ to be such that $f^{(n)}(s)=\operatorname{sgn} \lambda_{k}(t) \cdot\left|\lambda_{k}(t)\right|^{1 /(q-1)}$, while for $q=\infty$, we define $f$ such that $f^{(n)}(t)=\operatorname{sgn} \lambda_{k}(t)$. The fact that (4.10) is the best possible for $q=1$, can be proved as in [7, Thm 12].

\section{Mean value results}

In this section we consider mean value theorems involving $A_{k}$. Throughout the section we use this agreement that if $k \in\{1,2\}$, then $n \geq 3$. Further $k=1$ we assume that $\mathbf{x}$ and $\mathbf{p}$ satisfy the assumptions of Theorem 2.2 and for $k=2$ we assume that $\mathbf{x}$ and $\mathbf{p}$ satisfy the assumptions of Theorem 2.3 .

Theorem 5.1. Let $k \in\{1,2\}$ and let us consider $A_{k}$ as a functional on $C^{n}[a, b]$. If corresponding conditions from set $\{(2.13),(2.14)\}$ related to the fixed $k$, hold, then there exists $\xi_{k} \in[a, b]$ such that

$$
A_{k}(f)=f^{(n)}\left(\xi_{k}\right) A_{k}\left(f_{0}\right)
$$

where $f_{0}(x)=\frac{x^{n}}{n !}$.

Proof. Let us define functions

$$
F_{1}(x)=M f_{0}(x)-f(x)
$$

and

$$
F_{2}(x)=f(x)-L f_{0}(x)
$$

where $L$ and $M$ are minimum and maximum of the image of $[a, b]$, i.e.,

$$
F^{(n)}([a, b])=[L, M]
$$

Then $F_{1}$ and $F_{2}$ are $n$-convex. Hence $A_{k}\left(F_{1}\right) \geq 0$ and $A_{k}\left(F_{2}\right) \geq 0$ and

$$
L A_{k}\left(f_{0}\right) \leq A_{k}(f) \leq M A_{k}\left(f_{0}\right) .
$$

If $A_{k}\left(f_{0}\right)=0$, then the statement obviously holds.

If $A_{k}\left(f_{0}\right) \neq 0$, then $\frac{A_{k}(f)}{A_{k}\left(f_{0}\right)} \in[L, M]=f^{(n)}([a, b])$, so there exist $\xi_{k} \in[a, b]$ such that

$$
\frac{A_{k}(f)}{A_{k}\left(f_{0}\right)}=f^{(n)}\left(\xi_{k}\right) .
$$
result.

Applying Theorem 5.1 on function $\omega=A_{k}(h) f-A_{k}(f) h$, we get the following

Theorem 5.2. Let $k \in\{1,2\}$ and let us consider $A_{k}$ as a functional on $C^{n}[a, b]$. If corresponding conditions from set $\{(2.13),(2.14)\}$ related to the fixed $k$, hold, then there exists $\xi_{k} \in[a, b]$ such that

$$
\frac{A_{k}(f)}{A_{k}(h)}=\frac{f^{(n)}\left(\xi_{k}\right)}{h^{(n)}\left(\xi_{k}\right)}
$$

assuming that both the denominators are non-zero. 
Remark 5.3. If the inverse of $\frac{f^{(n)}}{h^{(n)}}$ exists, then from the above mean value theorems we can give generalized means

$$
\xi_{k}=\left(\frac{f^{(n)}}{h^{(n)}}\right)^{-1}\left(\frac{A_{k}(f)}{A_{k}(h)}\right) .
$$

Remark 5.4. Using the same method as in [7], we can construct new families of exponentially convex functions and Cauchy type means.

\section{References}

[1] Baloch, I.A., Pečarić, J., Praljak, M., Generalization of Levinson's inequality, J. Math. Inequal., 9(2015), 571-586.

[2] Butt, S.I., Mehmood, N., Pečarić, J., New generalization of Popoviciu type inequalities via new Green functions and Taylor's formula, submitted.

[3] Cerone, P., Dragomir, S.S., Some new Owstrowski-type bounds for the Čebyšev functional and applications, J. Math. Inequal., 8(2014), no. 1, 159-170.

[4] Fink, A.M., Bounds of the deviation of a function from its avereges, Czechoslovak Math. J., 42(117)(1992), 289-310.

[5] Khan, A.R., Latif, N., Pečarić, J.E., Exponential convexity for majorization, J. Inequal. Appl., 2012(2012): 105, 1-13.

[6] Khan, A.R., Pečarić, J., Lipanovic, M.R., n-Exponential convexity for Jensen-type inequalities, J. Math. Inequal., 7(2013), no. 3, 313-335.

[7] Khan, A.R., Pečarić, J.E., Praljak, M., Popoviciu type inequalities for $n$-convex functions via extension of Montgomery identity, submitted.

[8] Khan, A.R., Pečarić, J., Praljak, M., Varošanec, S., General Linear Inequalities and Positivity/ Higher order convexity, Monographs in inequalities 12, Element, Zagreb, 2017, pp. 269.

[9] Khan, A.R., Pečarić, J., Praljak, M., Varošanec, S., Positivity of sums and integrals of $n$-convex functions via the Fink identity, submitted.

[10] Khan, A.R., Pečarić, J.E., Varošanec, S., Popoviciu type characterization of positivity of sums and integrals for convex functions of higher order, J. Math. Inequal., 7(2013), no. 2, 195-212.

[11] Matić, M., Pečarić, J., Ujević, N., Some new Owstrowski-type bounds for the Čebyšev functional and applications, Computer. Math. Appl., 39(2000), no. 3-4, 161-175.

[12] Pečarić, J., On Jessenss inequality for convex functions, III, J. Math. Anal. Appl., 156(1991), 231-239.

[13] Pečarić, J., Perušić, A., Smoljak, K., Generalizations of Steffensen's inequality by AbelGontscharoff polynomial, Khayyam J. Math., 1(2015), no. 1, 45-61.

[14] Pečarić, J., Praljak, M., Witkowski, A., Linear operator inequality for n-convex functions at a point, Math. Ineq. Appl., 18(2015), 1201-1217.

[15] Pečarić, J., Proschan, F., Tong, Y.L., Convex Functions, Partial Orderings and Statistical Applications, Academic Press, New York, 1992.

[16] Popoviciu, T., Notes sur les fonctions convexes d'orde superieur III, Mathematica (Cluj), 16(1940), 74-86. 
[17] Popoviciu, T., Notes sur les fonctions convexes d'orde superieur IV, Disqusitiones Math., 1(1940), 163-171.

[18] Popoviciu, T., Les Fonctions Convexes, Herman and Cie, Editeurs, Paris 1944.

Asif R. Khan

Department of Mathematics, Faculty of Science,

University of Karachi,

University Road, Karachi-75270, Pakistan

e-mail: asifrk@uok.edu.pk

Josip Pečarić

RUDN University, Miklukho-Maklaya str. 6,

117198 Moscow, Russia

e-mail: pecaric@hazu.hr 